\title{
OBSERVATIONS OF SATURN'S INNER SATELLITES \\ DURING THE AUGUST 1995 RING-PLANE CROSSING
}

\author{
F. POULET, B. SICARDY, J.L. BEUZIT \\ Observatoire de Paris, Meudon, France
}

AND

P. PRADO

European Southern Observatory, Chile

\begin{abstract}
We present observations of Saturnian inner satellites, Janus, Epimetheus, Pandora and the new objects 1995S5 and 1995S6. Data were obtained with the COME-ON+ adaptive optics system mounted on the 3.6-m telescope of the European Southern Observatory, during the 10 August 1995 Earth crossing. During this time, the usually bright rings become faint, an ideal time to study the small inner satellites. Two unresolved objects have been detected. They have been identified with the objects 1995S5 and 1995S6, which have been detected on frames obtained with the Hubble Space Telescope, more than one day later. Astrometric reductions relative to the brighter satellites and the Cassini division yield planetocentric positions with accuracies of $0 \prime \prime 15$ and $0 ! 26$, respectively. These measurements, combined with the HST data, and the NIR photometric parameters point to the possible transient character of these objects, and provide a more accurate solution for the 1995S5 orbital parameters.
\end{abstract}

\section{Introduction}

Under typical conditions of illumination, the intense scattered light from both the globe of Saturn and its rings makes the observation of its small inner satellites extremely difficult. Only during the brief periods when the Earth passes through the ring plane, or is on the opposite side of the rings from the Sun, is the scattered light from the rings sufficiently reduced to permit observations of these objects. Janus (mean radius, $R=89 \mathrm{~km}$ ) and Epimetheus $(R=60 \mathrm{~km})$ are co-orbital satellites, and have been observed once since the Voyager 2 encounter. Pandora $(R=42 \mathrm{~km})$ and Prometheus

1. M. Wytrzyszczak, J. H. Lieske and R. A. Feldman (eds.),

Dynamics and Astrometry of Natural and Artificial Celestial Bodies, 525, 1997.

(C) 1997 Kluwer Academic Publishers. Printed in the Netherlands. 
( $R=50 \mathrm{~km}$ ) orbit on either side of the F ring, have poorly defined ephemerides because they are rarely observed. In 1995-96, there have been three Earth ring-plane crossings (22 May 1995, 10 August 1995 and 11 February 1996). During the August Earth ring-plane crossing, we identified Janus, Epimetheus and Pandora, and detected the new objects 1995S5 and 1995S6. Combining our data with the Hubble Space telescope data (Nicholson et al. 1996, hereafter N96), we derived orbital and photometrical parameters of these satellites.

\section{Observations and Data Reduction}

\subsection{OBSERVATIONS AND RING ASPECT}

During a 6-night period spanning the 10 August 1995 ring plane crossing, we obtained $60 \mathrm{sec}$ exposures of Saturn's rings using the COME-ON+ adaptive optics system with a short $\mathrm{K}$ filter, at the 3.6-m telescope of the European Southern Observatory (ESO La Silla). The atmospheric conditions (seeing of 1.5) prevented the achievement of diffraction-limited performances $\left(0{ }^{\prime \prime} 1\right)$, but we obtained images with an angular resolution as good as 0 "' 3 . The corrected images were recorded on the $256 \times 256$ SHARP II infrared camera with a 0.0505 pixel scale on the sky $(323 \mathrm{~km}$ at the distance to Saturn), leading to a total field of view of $12.9 \times 12^{\prime \prime} 9$. This field of view is not wide enough to contain the two ansae. By observing at near-infrared wavelengths, especially in the 2.0 to $2.33 \mu \mathrm{m}$ region, the scattered light from Saturn is greatly reduced by atmospheric methane and molecular hydrogen absorption. Only the nights of 9 and 10 August (before the ring-plane crossing) have been used to search for satellites. During this period, the elevation of the Earth above the ring plane went to -0.045 to -0.013 , while the rings were illuminated by the Sun with an incidence angle of +195 . The radial brightness profile of the dark side of the rings shows then the following: the $\mathrm{F}$ ring extends over $280000 \mathrm{~km}$ from West to East; no significant brightening is observed at the locations of the A and $B$ rings making easier the detection of faint objects, and a clear brightening due to the sunlight transmitted through the translucent $\mathrm{C}$ ring and Cassini Division are observed.

\subsection{IMAGE PROCESSING}

Standard infrared data reduction procedures were applied to each individual frame: sky subtraction, corrections of the pixel-to-pixel sensivity variations, cosmic rays elimination. A correction for specific detector electronic periodic patterns was also performed. The difficulties inherent in detecting small satellites result from the light scattered by the rings and the presence 


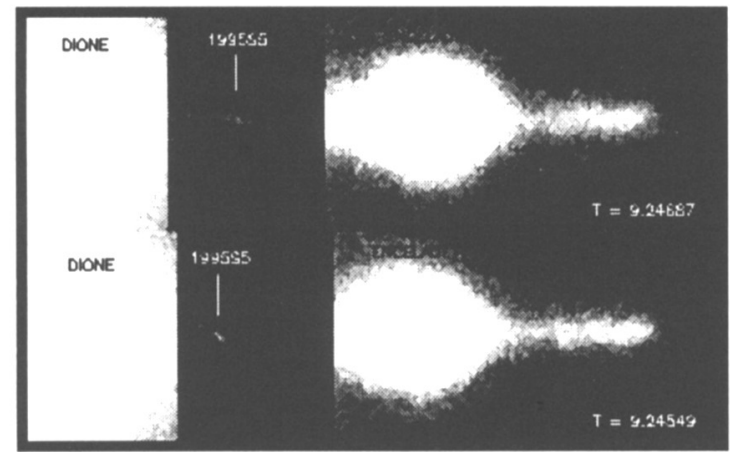

Figure 1. Sequence of two images of the east ansa obtained on 9 August showing the appearance and the orbital motion of 1995S5. The indicated time is in decimal day. Only part of a reference image has been subtracted around the object.

TABLE 1. Number of detections of objects.

\begin{tabular}{llllll}
\hline & Janus & Epimetheus & Pandora & 1995S5 & 1995S6 \\
\hline 09 August 1995 & $10(5)$ & $2(0)$ & $3(3)$ & $4(4)$ & $2(0)$ \\
10 August 1995 & $20(6)$ & 0 & $4(4)$ & 0 & $7(0)$ \\
\hline
\end{tabular}

In parentheses, number of detections used for the determination of orbital parameters.

of strong local gradient in the scattered light background due to the planet. We therefore experimented with several methods of subtracting the scattered light. The Janus and Epimetheus images are $\simeq 20$ pixels or $1^{\prime \prime}$ in size. Although close to the planet, these satellites have been easily detected. The situation was more complicated for the other satellites. A satisfactory solution to the scattered-light problem is to use either an earlier or later reference image in which no satellite appears, in order to subtract the scattered light in the images of interest. This procedure involves an astrometric shift and a photometric shift. In the first, we align the rings in the two images to take account of the motion of the telescope. Once the 2 images are superimposed, the ring's brightnesses are compared and adjusted for changing ring brightness when necessary. Using this shift and subtraction technique, we were able to detect Pandora in 3 frames on August $9^{\text {th }}$ and in 4 frames on the $10^{\text {th }}$, which constitues one of the first sightings with a ground-based telescope. Some exposures revealed the presence of objects, which cannot be explained by the known satellites ephemerides. On August $9^{\text {th }}$, one object is seen on 4 frames (Figure 1). We have identified this object with 1995S5 (Sicardy et al., 1996), detected on frames obtained with the HST (N96) more than one day later. The same day, two images show a big 
increase of the signal at the extremity of the east ansa. This phenomenon is also observed on August $10^{\text {th }}$ at the extremity of the east ansa (Sicardy et $a l ., 1995)$. No conspicuous point-like object is visible there on good quality images taken at other times. This object agrees with the new moon 1995S6 (N96). Our various sets of detections are summarized in Table 1.

\subsection{PLANET-SATELLITE ASTROMETRY}

We used the ephemeris files maintained at the Jet Propulsion Laboratory (Showalter, 1995). For each frame showing a clearly discernable image of a moon, the pixel coordinates plus those of the reference satellite (Tethys, Dione and/or Rhea), if available, were determined. The presence of strong local gradients in the scattered light background induces a systematic deviation of the satellite centroid measurements. We derived centroid positions by subtracting the local background in two rectangular strips around the rings, and then computing the center of light of the residual flux within a small aperture centered on the satellite. The centers of sources were determined by the centroiding routine of the IDL package for brighter satellites and "by eye" for fainter ones. The two methods agreed to within one pixel. The location of Cassini Division was used as a pointing reference in several images for which reference moons were unavailable. Because of the near edge-on aspect of the orbits, we fitted the separation of each body from Saturn's center in kilometers. The uncertainties assigned to these measurements depend on the method of measurement and systematic errors in the reference satellite ephemerides. The dark-side ring profiles extend to $140200 \mathrm{~km}$, the outer edge of the F ring. The study of these different profiles (Poulet and Sicardy, 1996) allows us to estimate uncertainties of $\pm 1000 \mathrm{~km}$ using a satellite as the reference and $\pm 1700 \mathrm{~km}$ using the Cassini division.

\section{Orbital and Photometric Parameters}

\subsection{ORBITAL FIT}

As indicated in Table 1, all detections could not be used for the orbital parameters determination, because no reference was available or the object like 1995S6 and Epimetheus were detected at greatest elongations, not improving the orbital fit. Combining the ESO data with the HST data (N96), the positions of Janus, Pandora and 1995S5 were fitted to a circular, noninclined orbit (Table 2). The fitted parameters are orbital radius $a$, the mean motion $n$ and the longitude $\lambda_{0}$ measured from the ascending node of Saturn's equatorial plane on the Earth's equatorial plane J2000 at reference time $t_{o}$ (defined to be 10 August 1995, 12:00 TDT at Saturn). The global 


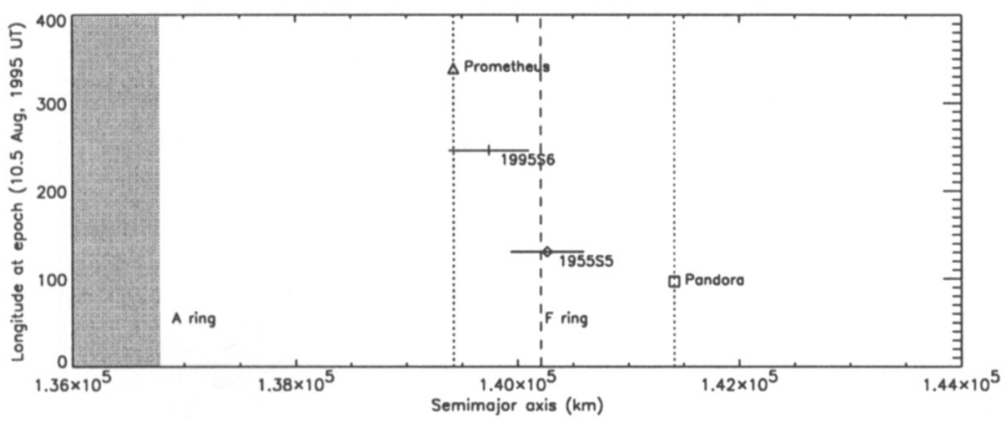

Figure 2. Longitudes of the new objects (1995S5-S6) at epoch vs their orbital radii. These are compared with the longitudes and radii of the $\mathrm{F}$ ring, Pandora and Prometheus. Prometheus and 1995S6 parameters is giving by N96. Hatched band is the outer A ring.

data recovering several revolutions obviously improve the mean motion determination. Hence, including the effect of Saturn's $J_{2}, J_{4}$ and $J_{6}$, the mean motion of 1995S5 yields a geometric orbital radius of $140268 \pm 440 \mathrm{~km}$, closer to the $F$ ring radius $(140209 \pm 4 \mathrm{~km})$ than the previous value derived from HST data alone. Figure 2 shows the relative location of the two new objects at our adopted epoch. Both 1995S5 and 1995S6 move close to the $F$ ring's semimajor axis.

TABLE 2. Orbital elements for Janus, Pandora and 1995S5 (Epoch JD=2 449940.0).

\begin{tabular}{llll}
\hline Parameters & Janus & Pandora & 1995S5 \\
\hline$a[\mathrm{~km}]$ & 151665 & 141293 & 139673 \\
$n[\mathrm{deg} / \mathrm{d}]$ & 519.13 & 570.13 & 581.69 \\
$\lambda_{0}$ & 35.61 & 96.53 & 130.57 \\
$\mathrm{rms}[\mathrm{km}]$ & 1748 & 1859 & 420 \\
$a_{\text {geom }}[\mathrm{km}]$ & 151289 & 142152 & 140268 \\
\hline
\end{tabular}

\subsection{PHOTOMETRIC CHARACTERISTICS}

Besides detection of satellites, the study of the ring profiles allows a photometric calibration. From the integrated flux, we estimated the albedo for the known satellites and the radius for the new objects, assuming a spherical shape and Janus's albedo. In Figure 3, we plot the integrated brightness (I/F) of all small objects visible in our images vs their mean radii. The 1995S5 and 1995S6 estimated radii are respectively of $37 \pm 4 \mathrm{~km}$ and $45 \pm 5 \mathrm{~km}$. These values are only meaningful if the new objects are satellites, but this interpretation leads to the following problem: the estimated 


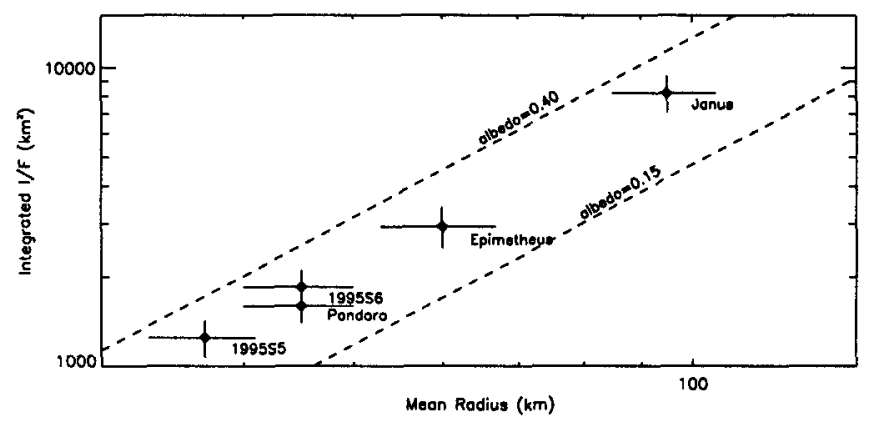

Figure 3. Integrated $\mathrm{I} / \mathrm{F}$ vs mean radius for the detected objects. Dashed lines are for geometric albedos of 0.15 and 0.40 .

radii are larger than those of $\operatorname{Pan}(R=15 \mathrm{~km})$ and Atlas $(R=15 \mathrm{~km})$, which were discovered in Voyager images.

\section{Conclusion}

Our observations have allowed observations of three inner satellites, Janus, Epimetheus and Pandora, and also the pre-discovery of two objects 1995S5 and 1995S6. These observations linked with the HST observations may be useful in answering questions about the evolution of Saturn's ring and particulary about the new "moons". What is the nature of $199555 / S 6$ ? These objects are observed near the orbit of the F ring, very close to a zone of dynamical instability. But none of them are retrieved in the May and November HST observations. Moreover, because of their equivalent radius of about $40 \mathrm{~km}$, they should have been seen by Voyager, if they are indeed moons. So, the most likely explanation is that they correspond to transient clumps or arcs embedded in the $F$ ring.

\section{References}

Nicholson, P.D., Showalter, M., Dones, L., French, R.G., Larson, S.M., Lissauer, J.J., McGhee, C.A., Seitzer, P., Sicardy, B., and Danielsen, G.E.: 1996, "Observations of Saturn's ring-plane crossings in August and November 1995", Science 272, 509-515.

Poulet, F. and Sicardy, B.: 1996, "The 1995 Saturn ring-plane crossings: Ring thickness and small inner satellites", in preparation.

Showalter, M.R.: 1995, "Saturn viewer, the ephemeris files", in: Planetary Data System's Rings Nodes (http//ringside.arc. nasa.gov/www/rpx/viewer/).

Sicardy, B., Poulet, F., Beuzit, J.L., and Prado, P.: 1995, "Satellites of Saturn", IAU Circ. 6269.

Sicardy, B., Poulet, F., Beuzit, J.L., and Prado, P.: 1996, "Satellites of Saturn", IAU Circ. 6395. 